\title{
Erratum to: Surgical unroofing of the optic canal and visual outcome in basal meningiomas
}

\author{
Giuseppe Mariniello • Oreste de Divitiis • \\ Giulio Bonavolontà • Francesco Maiuri
}

Published online: 7 October 2012

(C) Springer-Verlag Wien 2012

\section{Erratum to: Acta Neurochir}

DOI 10.1007/s00701-012-1485-Z

The original version of this article unfortunately contained mistake. Table 2 was incorrectly presented, correct table 2 is presented below:

The online version of the original article can be found at http://dx.doi.org/ 10.1007/s00701-012-1485-z.

G. Mariniello $\cdot$ O. de Divitiis $\cdot$ F. Maiuri

Department of Neurological Sciences, Section of Neurosurgery,

"Federico II" University School of Medicine,

Naples, Italy

G. Bonavolontà

Department of Ophthalmology, Section of Orbital Pathology,

"Federico II" University School of Medicine,

Naples, Italy

G. Mariniello $(\varangle)$

Università degli Studi di Napoli "Federico II",

Cattedra di Neurochirurgia, 5 Via S. Pansini,

Naples 80131, Italy

e-mail: giumarin@unina.it 
Table 2 Summary of the data of 46 clinoidal meningiomas

\begin{tabular}{|c|c|c|c|c|c|c|}
\hline \multicolumn{2}{|l|}{ Findings } & \multirow{2}{*}{$\begin{array}{l}\text { Overall (46 cases) } \\
11(24 \%)\end{array}$} & \multicolumn{2}{|c|}{ Group A (32 cases) } & \multicolumn{2}{|l|}{ Group B (14 cases) } \\
\hline \multirow[t]{3}{*}{ Al-Mefty type } & type I & & $8(25 \%)$ & & $3(21.5 \%)$ & \\
\hline & type II & $28(60.8 \%)$ & $20(62.5 \%)$ & & $8(57 \%)$ & \\
\hline & type III & $7(15.2 \%)$ & $4(12.5 \%)$ & & $3(21.5 \%)$ & \\
\hline \multirow[t]{3}{*}{ tumor size } & $<2.5 \mathrm{~cm}$ & $14(30.4 \%)$ & $9(28.1 \%)$ & & $5(35.7 \%)$ & \\
\hline & 2.5 to $4 \mathrm{~cm}$ & $18(39.2 \%)$ & $12(37.5 \%)$ & & $6(42.9 \%)$ & \\
\hline & $>4 \mathrm{~cm}$ & $14(30.4 \%)$ & $11(34.4 \%)$ & & $3(21.4 \%)$ & \\
\hline \multicolumn{2}{|c|}{ tumor extension into the $\mathrm{OC}$} & $10(22 \%)$ & $6(18.7 \%)$ & & $4(28.5 \%)$ & \\
\hline \multirow{3}{*}{\multicolumn{2}{|c|}{ early ON visualization }} & & $<2.5 \mathrm{~cm}$ & $7 / 9$ & $<2.5 \mathrm{~cm}$ & all \\
\hline & & & 2.5 to $4 \mathrm{~cm}$ & $2 / 12$ & 2.5 to $4 \mathrm{~cm}$ & all \\
\hline & & & $>4 \mathrm{~cm}$ & none & $>4 \mathrm{~cm}$ & all \\
\hline \multicolumn{2}{|c|}{ early ICA visualization } & & \multicolumn{2}{|c|}{$\begin{array}{l}5(15.6 \%) \\
(<2.5 \mathrm{~cm} ; \text { types II and III })\end{array}$} & $14(100 \%)$ & \\
\hline \multicolumn{2}{|c|}{ complete tumor resection } & $39(84.8 \%)$ & \multicolumn{2}{|c|}{$26(81 \%)$} & $13(93 \%)$ & \\
\hline \multicolumn{2}{|c|}{ preoperative visual symptoms } & $30(65.2 \%)$ & $20(62.5 \%)$ & & $10(71.4 \%)$ & \\
\hline \multirow[t]{3}{*}{ visual outcome } & improved & $17(56.7 \%)$ & $9(45 \%)$ & & $8(80 \%)$ & \\
\hline & stable & $12(40 \%)$ & $10(50 \%)$ & & $2(20 \%)$ & \\
\hline & worsened & $1(3.3 \%)$ & $1(5 \%)$ & & - & \\
\hline
\end{tabular}

$O C$ optic canal; $O N$ optic nerve; $I C A$ internal carotid artery 